\title{
Electronic Detection of Escherichia coli 0157：H7 Using Single-Walled Carbon Nanotubes Field-Effect Transistor Biosensor
}

\author{
Xiaoxian Zhang ${ }^{1}$, Dongwei Wang ${ }^{1}$, Danna Yang ${ }^{1}$, Sai $\mathrm{Li}^{{ }^{*}}$, Zhiqiang Shen ${ }^{2}$ \\ ${ }^{1}$ School of Chemical Engineering, Sichuan University, Chengdu, China \\ ${ }^{2}$ Academy of Military Medical sciences, Chinese PLA Center for Disease Control \& Prevention, Tianjin, China \\ Email: "lisai@scu.edu.cn
}

Received 2012

\begin{abstract}
Field effect transistors (FET) based on Single-Walled Carbon Nanotubes (SWNTs) become the hot topic in fields of nano-electronic, clinical diagnostics, environmental testing etc. in recent years. In this paper, we reported a simple, scalable way to enrich semiconducting SWNTs by using HNO3/H2SO4. Then carbon nanotube field-effect transistors (CNTFET) biosensor was fabricated with the enrichment SWNTs for Escherichia coli O157 : H7 detection. The response of each CNTFET was monitored in real time before and after introduction of the Escherichia coli O157 : H7 at various concentrations. The results show that CNT-FET biosensors we fabricated are sensitive to change of concentration of solution and response time is really short.
\end{abstract}

Keywords: Single-walled Carbon Nanotubes (SWNTs); Field Effect Transistors; Biosensor; Escherichia Coli O157 : H7

\section{Introduction}

Biosensor plays an important role in many areas of environmental monitoring, food analysis and life sciences. But traditional biological sensing techniques require multiple reagents, signal amplification, and complex data analysis [1]. Because SWNTs have outstanding properties such as possible biocompatibility, size compatibility and sensitivity towards minute electrical perturbations [2], it has a great potential for biosensing applications. Besides, CNTFET fabricated with semiconducting SWNTs can operate at room temperature, reagentless and label-free.

CNTFET has been used as biosensors to detect target biomolecules such as virus [3], bacterium [4,5], DNA [6] and enzymes [7]. Star et al. [8] have fabricated CNTFET devices sensitive to streptavidin by using individual biotin-functional- ized CNTFET. Li et al. [9] reported the complementary detection of prostate-specific antigen (PSA) by using an anti-PSA monoclonal antibody CNTFET. The mechanism of detection of biomolecules is still controversial. There are two major explains: charge-transfer mechanism (electrostatic gating) [10] and Schottky-Barrier modulation effect [11].

But there's a hurdle to the widespread application of CNTFET. SWMTs synthesized are the mixture of semiconducting SWNTs (sem-SWNTs) and metallic SWNTs (met-SWNTs). The undesired met-SWNTs will hinder effective electronic switching. Therefore, it's essential to enrich or separate semSWNTs from met-SWNTs. Many proposed methods were put forward, such as dielectrophoresis [12], aromatic molecules [13] and DNA wrapped [14]. But there's not a scalable, nondestructive, iteratively repeatable and affordable sorting strategy

*Corresponding author. emerged [15].

In this paper, we separate metallic from semiconducting nanotubes by using $\mathrm{HNO}_{3} / \mathrm{H}_{2} \mathrm{SO}_{4}$. Then we fabricate CNTFET biosensor with treated SWNTs by applying AC dielectrophoresis [16] method. The CNTFET biosensor was functionalized with linker molecule and the corresponding antibody. The binding of the target E. coli O157 : H7 onto the receptor detected by monitoring the electronic properties between source and drain electrodes. We hope our work could contribute to fast detection and comprehend of mechanism.

\section{Materials and Methods}

\subsection{Materials}

E. coli O157 : H7 antibody and E. coli O157 : H7 were supplied by Academy of Military Medical sciences, Chinese PLA Center for Disease Control \& Prevention. 1-pyrenebutanoic acid succinimidyl ester (PASE) was purchased from SigmaAldrich. The reagents Dimethyl Formamide was purchased from Kelong Chemical Company. All chemicals were used without purification. CVD SWCNTs were produced by Chengdu Organic Chemistry Research Center.

\subsection{Preparation of CNTFET Biosensor}

The procedure of separate metallic from semiconducting nanotubes and fabricate CNTFET can be seen in our precious works $[17,18]$.

Immerse CNT channels in a $6 \mathrm{mM}$ solution of PASE in dimethyl formamide solution in room temperature overnight. Then washed it with deionized water and blown dry with nitrogen. We can get a PASE-CNTFET. Secondly, the channels of 
PASE-CNTFET were submerged in a $4.7 \mathrm{mg} / \mathrm{mL}$ solution of E. coli O157 : H7 antibody in phosphate buffer solution (PBS) overnight. The device surface was then thoroughly rinsed with deionized water to remove the unreacted antibodies and dried under a stream of nitrogen.

\subsection{Detection of E. coli 0157 ：H7}

Immerse channels of antibody-CNTFET biosensors in solutions of E. coli O157 : H7 at different concentrations of $8.2 \times 10^{2}$, $8.2 \times 10^{4}, 8.2 \times 10^{6}, 8.2 \times 10^{8} \mathrm{CFU} / \mathrm{mL}$ in PBS. The electrical properties of the CNTFETs were measured in real time using a semiconductor parameter analyzer. We also detected antibody-CNTFET which was not modified with PASE as a reference to explore the impact of linker molecule.

\subsection{Characterization Techniques}

The SWNTs pattern on the substrate was observed by a Scanning Electron Microscopy (FE-SEM, Hitachi S4800) to evaluate the morphology of the samples. The CNTFETs have been characterized at room temperature using an Agilent-4155C semiconductor analyzer. Raman spectra were measured with a HORIBA LabRAM HR Ramanscope.

\section{Results and Discussion}

\subsection{Enrichment of Sem-SWNTs}

By contrast with Kataura plot, we can know the regions of sem-SWNTs and met-SWNTs response relativity [19]. With $532 \mathrm{~nm}$ laser, the peaks with wavenumbers $215-292 \mathrm{~cm}^{-1}(0.8-$ $1.1 \mathrm{~nm})$ are due to met-SWNTs while those $135-215 \mathrm{~cm}^{-1}$ $(1.1-1.82)$ and $293-330 \mathrm{~cm}^{-1}(0.74-0.8 \mathrm{~nm})$ are due to semSWNTs. As shown in Figure 1(a), we can see an obvious increase of sem-peaks around 160, 200 and $300 \mathrm{~cm}^{-1}$ after $\mathrm{HNO}_{3} / \mathrm{H}_{2} \mathrm{SO}_{4}$ treatments for $24 \mathrm{~h}$, whereas the met-peaks were almost maintain same as pristine SWNTs.

It may caused by charge transfer from SWNTs to $\mathrm{NO}_{2}{ }^{+}$. It's well known that mixing $\mathrm{HNO}_{3}$ and $\mathrm{H}_{2} \mathrm{SO}_{4}$ yields a high production rate of $\mathrm{NO}_{2}^{+}$[20]. $\mathrm{NO}_{2}^{+}$as an electron less structure can selectivity attack met-SWNTs because they have more available charge density at the Fermi level than sem-SWNTs, which induced the selective removal of met-SWNTs.

Figure 1(b) demonstrates D and G band of SWNTs before and after treatment. There's an obvious drop in D band around $1450 \mathrm{~cm}^{-1}$, which means a decrease of disorder SWNTs, in other words, treatment with $\mathrm{HNO}_{3} / \mathrm{H}_{2} \mathrm{SO}_{4}$ can purificate SWNTs as well as enrich sem-SWNTs.

\subsection{Device Layout and Electrical Characterization of CNTFET}

Figure 2 shows the SEM image of electrode after SWNTs assembly on Au electrodes. A lot of SWNTs bundles are arranged between the sources and drain electrodes. Figure 3 reveals the output characteristics of the CNTFET device fabricate with enrichment of sem-SWNTs. From the transfer characteristics, we can see that the drain current $I_{d}$ decreases with increasing gate voltage $\mathrm{Vg}$ in the range between $-10 \mathrm{~V}$ and $+10 \mathrm{~V}$. This result indicated that CNT-FET showed p-type characteristics in the blank PBS buffer.

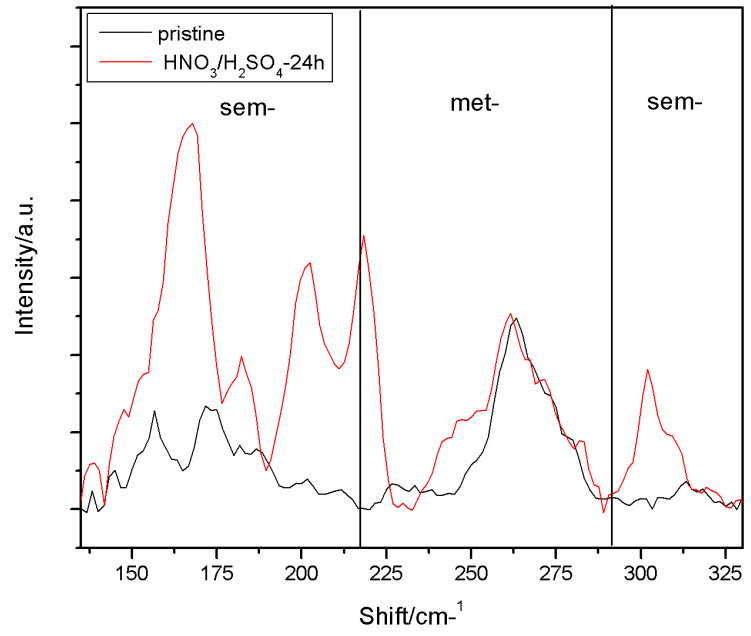

(a)

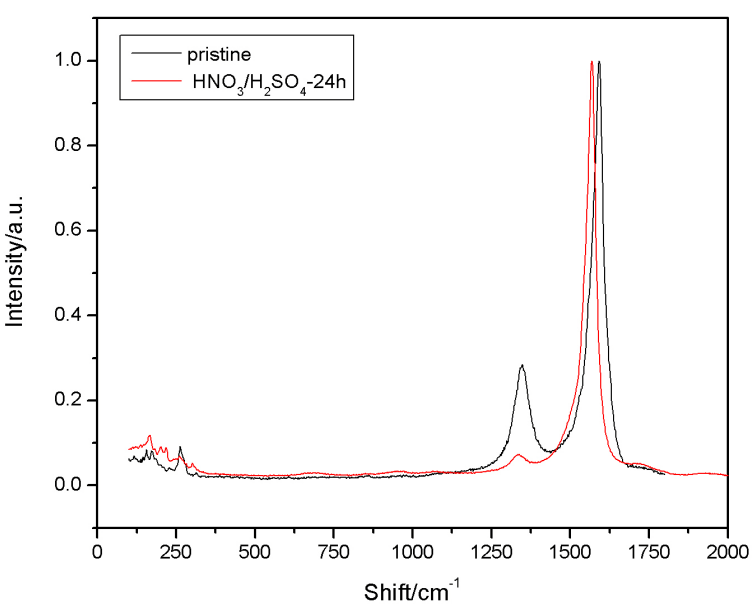

(b)

Figure 1. Raman spectra of pristine SWCNTs and $\mathrm{HNO}_{3} / \mathrm{H}_{2} \mathrm{SO}_{4}(2: 1)$ treatment for $24 \mathrm{~h}$ samples in RBM regoin (a) and $\mathrm{G}$ bands(b) under $532 \mathrm{~nm}$ (data normalized with with peak at $260 \mathrm{~cm}^{-1}$ and G band reference).

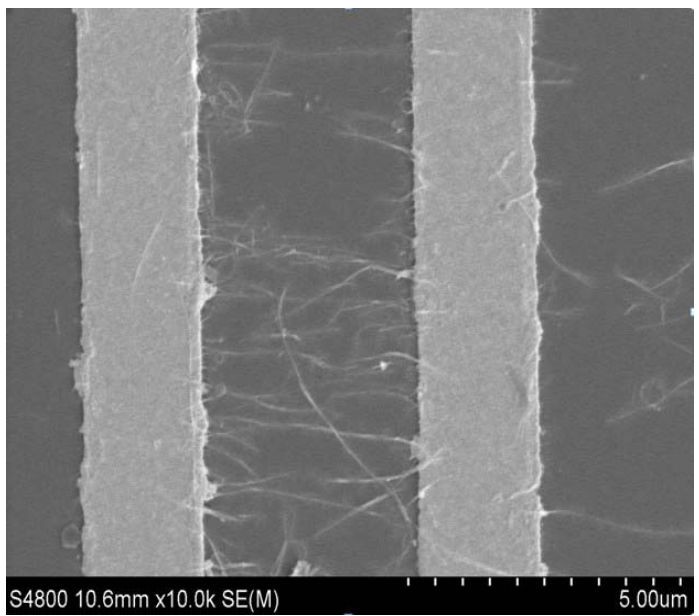

Figure 2. SEM image of CNTFET. 


\subsection{Detection of the E. coli 0157 : H7 on CNTFET Modified by Linker and Antibody}

It's a common way to functionalize SWNTs by using corresponding receptor molecules as recognition layer to realize specific detection of biomolecules. We detected E. coli O157 : H7 using CNTFET biosensors, in which CNTFET channels were modified with E. coli O157 : H7 antibody. Linker bifunctional molecule PASE can attach with antibody and SWNTs respectively, playing a role as the bridge medium. The illustration for fabrication of CNTFET immune biosensor was showed in Figure 4.

Resistance-voltage curves of CNTFET were demonstrated in Figure 5 and Figure 6. Figure 5 shows that after modified with PASE, the antibody-CNTFET biosensor causes a significant decrease in the source-drain resistance contrast with the pristine CNTFET. The significant change means E. coli $\mathrm{O} 157$ : H7 could be captured by the antibody, then resistance change follows. Figure 6 shows Resistance-voltage curve of CNTFET after introduction of $\mathrm{O} 157$ : H7 into antibody-CNTFET without PASE modified has slight change, which means CNTFET without modified by linker could not attach effective antibody to capture E. coli O157 : H7.

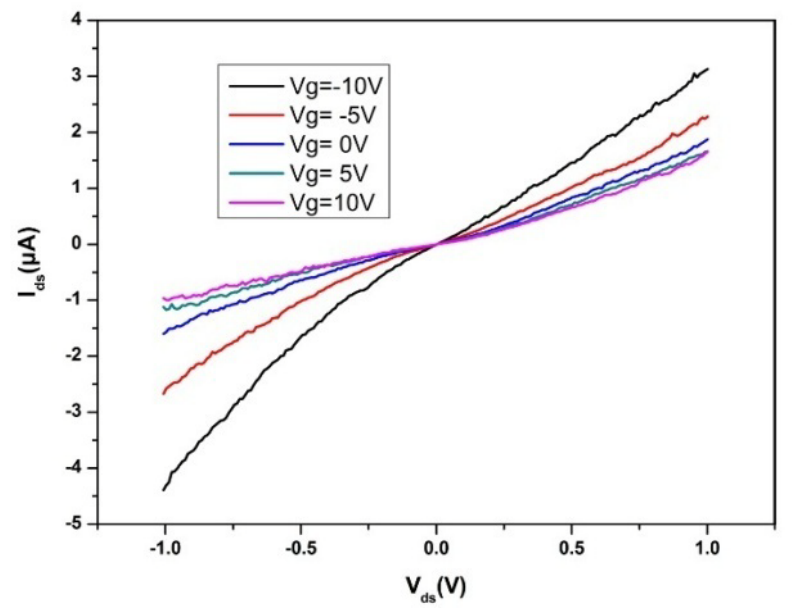

Figure 3. Id -Vds curves of a CNTFET measured at room temperature. $(\mathrm{Vg}=-10$ to $10 \mathrm{~V}$ and steps are $5 \mathrm{~V})$.
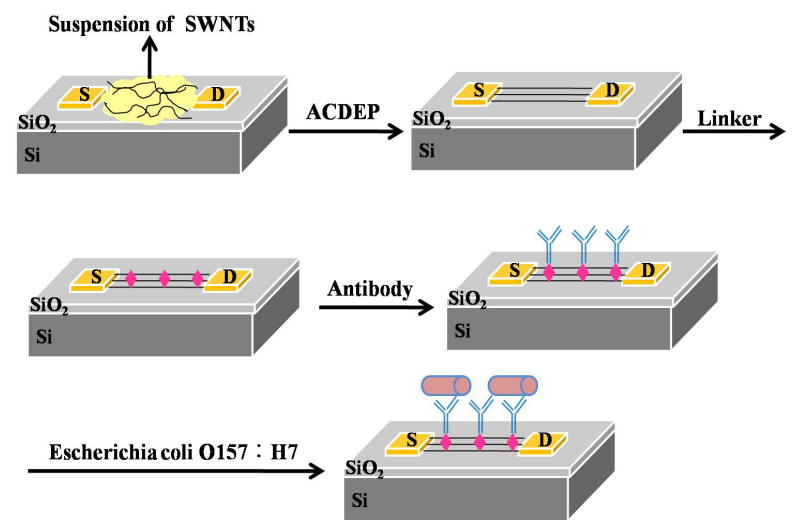

Figure 4. Schematic illustration for fabrication of CNTFET immune biosensor.

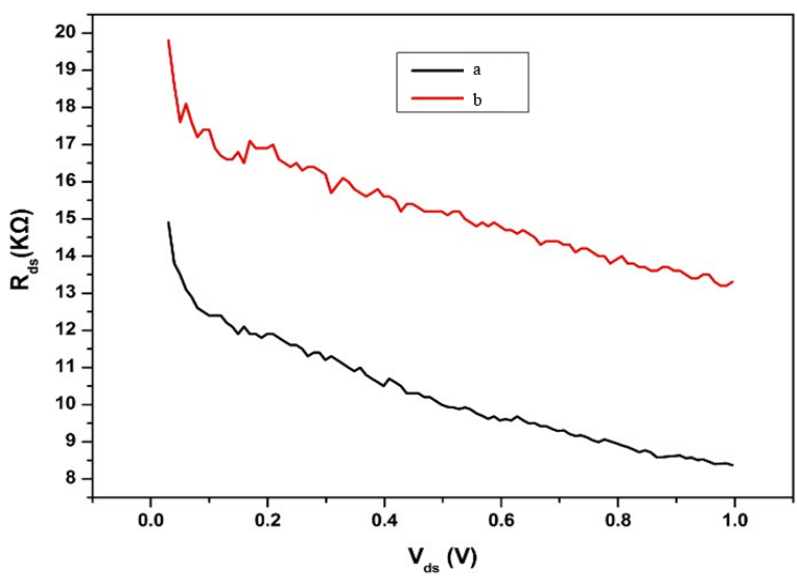

Figure 5. Resistance of Voltages of antibody-CNTFET after introduction of 0157 : $H 7$ into antibody- CNTFET with PSE. a: PASE-CNTFET, b: pristine CNTFET.

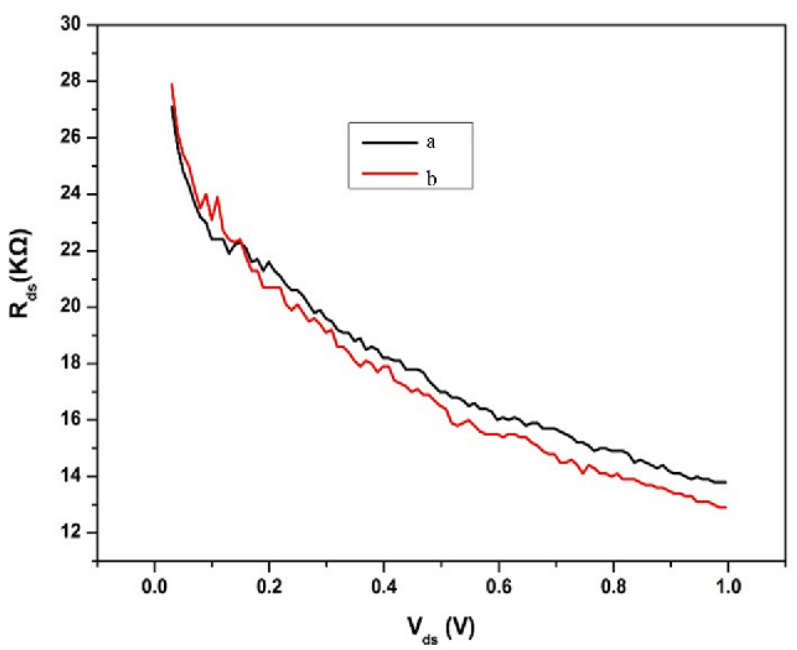

Figure 6. Resistance of Voltages of antibody-CNTFET after introduction of 0157 : $\mathrm{H7}$ into antibody-CNTFET without PASE.a:CNTFET without PASE, b:pristine CNTFET.

After measuring of electrical properties of the CNTFET biosensors, we washed the device surface with deionized water several times and dried under a stream of nitrogen. Then we observed using SEM to confirm whether E. coli O157 : H7 were attached on channel of CNTFET or not.

Figure 7 shows SEM results. CNTFET after PASE modified could attach E. coli O157 : H7 (Figure 7(a)) whereas those CNTFET without PASE could not catch the E. coli O157 : H7 (Figure 7(b)).

Combine R-V Curves and SEM results, we can get the conclusion that the CENFET biosensors could realize the effective detection of E. coli O157 : H7. Only the CNTFET modified by linker PASE and antibody have adsorption and response effect on E. coli O157 : H7. The linker PASE plays an important role to immobilize nanotubes and antibody. It's due to the special structure of PASE. It has two functional groups pyrene and succinimidyl ester. Pyrene moiety could functionalize with the SWNTs by $\pi-\pi$ stacking and succinimidyl ester could react with -NH2 in antibody to be a covalent bond. 


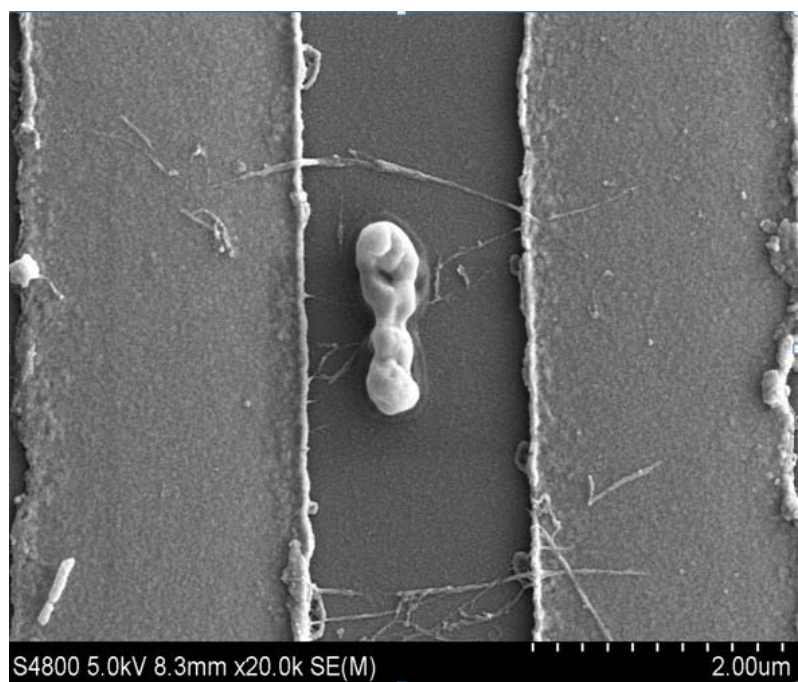

(a)

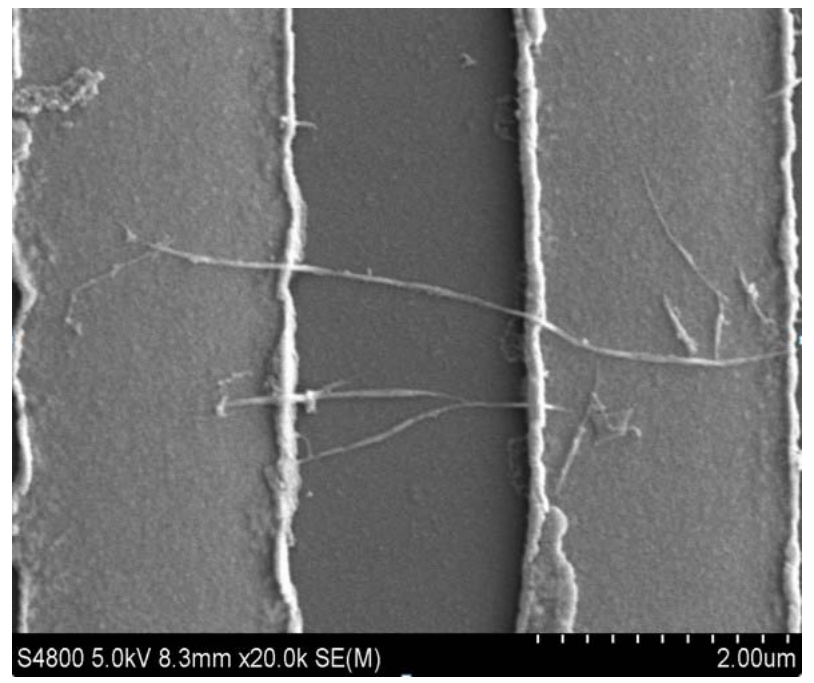

(b)

Figure 7. SEM images of CNTFET after introduction of $0157:$ H7 (a) PASE-CNTFET (b) CNTFET without PASE.

Time dependence of resistance of CNTFET after introduction of $\mathrm{O} 157$ : $\mathrm{H} 7$ at different concentration into antibodyCNTFET was showed in Figure 8. Solution of concentrations $8.2 \times 10^{2}, 8.2 \times 10^{4}, 8.2 \times 10^{6}, 8.2 \times 10^{8} \mathrm{CFU} / \mathrm{mL}$ of E. coli $\mathrm{O} 157$ : H7 in PBS was dropped in channels of antibody-CNTFET biosensors. The electrical properties of the CNTFETs were measured in real time using a semiconductor parameter analyzer. Resistance increases sharply to the maximum after dropped the solution. Then it decreases rapidly in around 50s to a relative stable level. Same trend occurred in different concentrations, and maximum of resistance increased from $84 \mathrm{k} \Omega$ to $110 \mathrm{k} \Omega$ along with the increase of concentration of E. coli O157 : H7 solution. The main reason is more E. coli O157 : H7 could be captured by antibodies on CNTFET along with the increase of concentration, then resistance between source and drain electrode rises consequently.

The changes of resistance indicates the possibility of CNTFET biosensor to detect E. coli O157 : H7, and concentra-

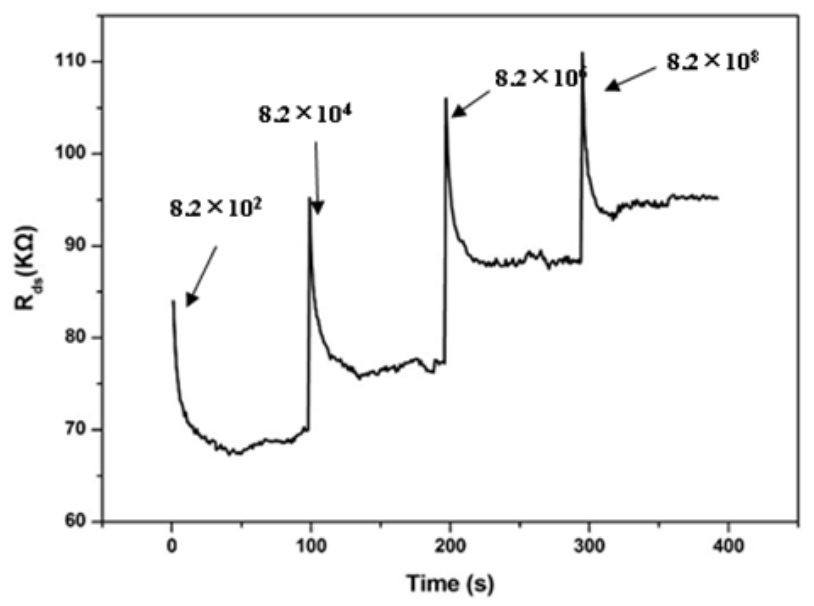

Figure 8. Time dependence of resistance of CNTFET after introduction of $0157: \mathrm{H} 7$ at different concentration into antibody-modified CNTFET.

tions as low as $8.2 \times 10^{2} \mathrm{CFU} / \mathrm{mL}$ can be readily detected with response times of about $50 \mathrm{~s}$.

\section{Conclusion}

In this paper, we successfully fabricated CNTFET biosensor with enriched semiconducting SWNTs by using a simple, scalable way of $\mathrm{HNO}_{3} / \mathrm{H}_{2} \mathrm{SO}_{4}$. From SEM images and R-V curves, we confirmed the effect of linker PASE. It can functionalize with the SWNTs as well as the antibody. Then solutions of concentrations $8.2 \times 10^{2}, 8.2 \times 10^{4}, 8.2 \times 10^{6}, 8.2 \times 10^{8} \mathrm{CFU} / \mathrm{mL}$ of E. coli O157 : H7 were detected with antibody-CNTFET biosensor. There's an obvious increase of resistance between source and drain with increase of concentration. We can draw a conclusion that the CNTFET biosensors we fabricated are sensitive to change of concentration of solution. The strategy can be applied to general antibody-based detection schemes for detecting concentration of target biomolecules.

\section{Acknowledgements}

This research was supported by NSFC (20805033; 30901199), SRF for ROCS, SEM (2008890-19-9).

\section{REFERENCES}

[1] Gruner G, "Carbon nanotube transistors for biosensing applications,” Anal. Bioanal. Chem., vol.384, pp. 322-335, 2006.

[2] Allen B L, Kichambare P D, Star A, "Carbon nanotube field-effect-transistor-based biosensors,”. Adv Mater., vol. 19, pp. 1439-1451, 2007.

[3] Oh J, Yoo S, Chang Y W et al. "Carbon nanotube-based biosensor for detection hepatitis B," Current Applied Physics, vol. 9, pp. E229-E231, 2009.

[4] Raquel A. Villamizar1, Alicia Maroto and F. Xavier Rius, "Improved detection of Candida albicans with carbon nanotubefield-effect transistors," Sensors and Actuators B. vol. 136, pp. 451-457, November 2009.

[5] Villamizar R A., Maroto A, Riusa F.X, Inza I and Figueras M J., "Fast detection of Salmonella Infantis with carbon nanotube field effect transistors,” Biosens. Bioelectron.. vol. 24, pp. 279-283, 
April 2008.

[6] Martinez M T, Tseng Y C, Ormategui N et al. "Label-free DNA biosensors based on functionalized carbon nanotube field effect transistors,” Nano Letters, vol. 9, pp.530-536,2009.

[7] Besteman K, Lee J O, Wiertz F G M, Heering H A and Dekker C, "Enzyme-coated carbon nanotubes as single-molecule biosensors,” Nano Letters, vol. 3, pp. 727-730, 2003.

[8] Alexander Star,Jean-Christophe P. Gabriel, Keith Bradley, and George Gru1ner, "Electronic detection of specific protein binding using nanotube FET devices,”Nano Letts. vol. 3, pp. 459-463, February 2003.

[9] Li C, Curreli M, Lin H, Lei B, Ishikawa F.N., Datar R, et al, "Complementary detection of prostate-specific antigen using ln2O3 nanowires and carbon nanotubes,” J. Am. Oil Chem. Soc., vol. 127, pp. 12484-12485, 2005.

[10] Bradley K, Briman M, Star A and Gruner G, "Charge transfer from adsorbed proteins,” Nano Letters, vol. 4, pp. 253-256,2004.

[11] Chen C, Zhang W, Zhao B and Zhang Y, "Investigation of Schottky-Barrier carbon nanotube field-effect transistor by an efficient semi-classical numerical modeling," Phys. Lett. A, vol. 374, pp. 309-312, 2009.

[12] Ralph Krupke, Frank Hennrich, Hilbert v. Lohneysen and Manfred M.Kappes, "Separation of metallic from semi-conducting single walled carbon nanotubes,” Science, vol. 301, pp. 344-347, July 2003

[13] Douglas R. Kauffman, Oleksandr Kuzmych and Alexander Star, "Interactions between single-walled carbon nanotubes and tetra- phenyl metalloporphyrins: correlation between spectroscopic and FET measurements,” J. Phys. Chem. C, vol. 111, pp. 3539-3543, 2007.

[14] Zheng M, Jagota A, Semke E D., Bruce A. Diner, Robert S. Mclean, Steve R. Lustig, et al., "DNA-assisted dispersion and separation of carbon nanotubes,” Nat. Mater, vol. 2, pp. 338-342, April 2003

[15] Mark C. HersaM, "Progress towards monodisperse single-walled carbon nanotubes,” Nature Nanotechnology, vol. 3, pp.387-394, July 2008.

[16] Stokes P, Silbar E, Zayas Y M., and Khondaker S I.,"Solution processed large areafield effect transistors from dielectroph oreticly aligned arrays of carbon nanotubes,” Appl. Phys. Lett., vol 94, pp. 113104-1-3, March 2009.

[17] Zhang X, Wang D, Yang D, Li S and He Y, "Field-effect Transistor Biosensor Fabricated With Selective Enrichment of Semiconducting Single-Walled Carbon Nanotubes,” ICoBE, pp. 27-28, February 2012.

[18] Yang D, Wang L, Zhao Q and Li S, "Fabrication Of Single-Walled Carbon Nanotubes (SWNTs) Field-Effect Transistor (FET) Biosensor,” BMEI 3rd,vol. 4, pp.1482-1485, 2010.

[19] H.Kataura, Y. Kumazawa, Y. Maniwa, I. Umezu, S. Suzuki, Y. Ohtsuka, et al,, "Optical properities of single-walled carbon nanotubes," Synth.Met. vol. 103, pp. 2555-2558, 1999.

[20] Francis A. C and Richard J. S, Advantaged Organic Chemistry; McGraw-Hill: New York, 1996. 